\title{
[18F]FDG PET-CT in Patients With DLBCL Treated With CAR-T Cell Therapy: A Practical Approach of Reporting Pre- and Post-treatment Studies
}

\section{Dan Cohen}

Tel Aviv Sourasky Medical Center https://orcid.org/0000-0001-8804-4806

\section{Efrat Luttwak}

Tel Aviv Sourasky Medical Center Ofrat Beyar-Katz

Tel Aviv Sourasky Medical Center Shir Hazut Krauthammer

Tel Aviv Sourasky Medical Center Yael Bar-On

Tel Aviv Sourasky Medical Center

Odelia Amit

Tel Aviv Sourasky Medical Center

\section{Ronit Gold}

Tel Aviv Sourasky Medical Center

\section{Chava Perry}

Tel Aviv Sourasky Medical Center

Irit Avivi

Tel Aviv Sourasky Medical Center

\section{Ron Ram}

Tel Aviv Sourasky Medical Center

Einat Even-Sapir ( $\boldsymbol{\nabla}$ evensap@tlvmc.gov.il )

Tel Aviv Sourasky Medical Center

\section{Research Article}

Keywords: CAR-T, DLBCL, PET-CT, Deauville, SUVmax

Posted Date: July 30th, 2021

DOl: https://doi.org/10.21203/rs.3.rs-749949/v1 
License: (c) (i) This work is licensed under a Creative Commons Attribution 4.0 International License. Read Full License

Version of Record: A version of this preprint was published at European Journal of Nuclear Medicine and Molecular Imaging on September 4th, 2021. See the published version at https://doi.org/10.1007/s00259-021-05551-5. 


\section{Abstract}

Purpose: The introduction of CD19-specific chimeric antigen receptor T-cell therapy (CAR-T) for treatment of relapsed/refractory diffuse large $B$ cell lymphoma (R/R DLBCL) gives hope to patients with otherwise dismal prognosis. Therapy outcomes depend, however, on selection of patients and on accurate early identification of non-responders. Patients treated with CAR-T usually undergo [ $\left.{ }^{18} \mathrm{~F}\right] \mathrm{FDG}$ PET-CT at time of decision (TD), time of CAR-T transfusion (TT), one month (M1) and three months (M3) post therapy. The purpose of the current study was to identify the specific parameters that should be addressed when reporting PET-CT studies in the clinical setting of CAR-T therapy.

Methods: A total of 138 PET-CT scans (30 TD, 42 TT, 44 M1, 22 M3) of 48 patients treated with CAR-T were included. SUVmax, TMTV, TLG were calculated in all scans. Response was assessed using Deauville scale and $\triangle S U V m a x$ method. Overall survival (OS) was the primary endpoint. Median follow-up was 12.8 (IQR 6.4-16.0) months from CAR-T infusion.

Results: In a univariate analysis, TD-SUVmax $>17.1$ and TT-SUVmax $>12.1$ were associated with shorter OS $(\mathrm{PV}<0.05)$. In a multivariate analysis, three factors were significantly associated with shorter OS: TDSUVmax > 17.1 (HR 10.3; PV<0.01), LDH > 450 U/I (HR 7.7; Pv<0.01) and ECOG score > 1 (HR 5.5; $\mathrm{PV}=0.04)$. Data from TD and TT PET-CT scans were not predictive of toxicity. On M1-PET-CT, patients with Deauville score $>3$ had significantly shorter OS (median 7.9 months, versus not reached, $\mathrm{PV}<0.01$ ). $\triangle$ SUVmax $\leq 66 \%$ on M1-PET-CT predicted shorter OS when comparison of M1-SUVmax was made to TDSUVmax (Pv=0.02) and not to TT-SUVmax (Pv=0.38).

Conclusion: Pre-treatment SUVmax may guide patient selection for CAR-T therapy. On M1-PET-CT, Deauville score and $\triangle S U V m a x$ from TD may identify early therapy failure. These parameters are easy to obtain and should be included in the PET-CT report.

\section{Introduction}

Overall survival (OS) of patients with relapsed/refractory diffuse large $B$ cell lymphoma (R/R DLBCL) who failed at least two treatment regimens is short, between 4.4-6.3 months [1,2]. This group of patients had limited treatment options prior to the recent approval of two commercially available CD19-specific chimeric antigen receptor T-cell (CAR-T) therapy, Axicabtagene ciloleucel (axi-cel) and Tisagenlecleucel (tisa-cel) [3-5].

Data from pivotal trials suggest durable remission in $30-40 \%$ of patient with R/R DLBCL treated with CAR-T therapy [4-5]. However, this therapy is also associated with toxicity, including cytokine release syndrome (CRS) and immune effector cell-associated neurotoxicity syndrome (ICANS), which can be lifethreatening [6-8]. Benefit from CAR-T relies on selection of patients and reduction of toxicity associated with the therapy. Non-responders should be identified as early as possible after CAR-T infusion so alteration of treatment approach may be considered. 
${ }^{18}$ F-Fluorodeoxyglucose positron emission tomography - computed tomography ([ $\left.{ }^{18} \mathrm{~F}\right] \mathrm{FDG}$ PET-CT) plays a key role in the management of patients with DLBCL and has been shown to predict outcome at specific time points in the earlier course of the disease [9-18]. In the clinical setting of CAR-T therapy, patients undergo $\left[{ }^{18}\right.$ F]FDG PET-CT at four relevant time points. Two scans are performed before CAR-T infusion: time of decision (TD) PET-CT, based on which the clinician select CAR-T as the next therapy, and time of transfusion (TT) PET-CT, performed immediately prior to the infusion of CAR-T. Post-therapy, two PET-CT scans are performed to monitor response to therapy: one month (M1) PET-CT and three months (M3) PET-CT.

Several previous studies focused on the role of PET-CT in the mentioned time points [19-23]. In a model built by Vercellino et al for prediction of early progression, total metabolic tumor volume (TMTV) was the only PET parameter assessed. The model identified high TMTV values obtained on TD and TT scans as risk factors for disease progression within one month after therapy [24]. Wang et al found an association between TT-TMTV and severe CRS [20], while in a recent study by lacoboni et al higher TT-TMTV showed no association with CRS but was associated with lower PFS [21].

In a busy clinical setting, TMTV is not routinely calculated and included in PET-CT reports. Monitoring response to first line therapy in DLBCL on $\left[{ }^{18} \mathrm{~F}\right] \mathrm{FDG}$ PET-CT is assisted by using more practical methods: the Deauville five-point scale or measurement of $\Delta S U V \max$. The Deauville five-point scale is based on a visual comparison between the uptake of lymphoma tissue and that of the liver and mediastinum, with a cut-off for the definition of an unfavorable response as an uptake greater than that of the liver [24]. Using the $\triangle S U V$ max method, the maximum standardized uptake value (SUVmax) of the "hottest" tumor lesion at two PET studies is compared. An unfavorable response is defined when SUVmax reduction is less than or equal to $66 \%$, a cut-off that has been confirmed in several studies [25-28]. The role of both methods has not yet been determined in R/R DLBCL patients treated with CAR-T.

In the current study we aimed to provide a practical guide for imaging interpretation of $\left[{ }^{18} \mathrm{~F}\right] \mathrm{FDG}$ PET-CT performed before and after CAR-T therapy. Specifically, we aimed to identify pre-CAR-T PET-CT parameters that may assisst in patient selection, and post-CAT-T PET-CT parameters that identify early CAR-T failure.

\section{Methods}

\section{Patient Population.}

After receiving the consent of the institutional ethical committee, we retrospectively screened the medical records of all patients that met the following inclusion criteria: (i) over 18 years old (ii) treated with CD19targeted CAR-T for R/R DLBCL (iii) clinically evaluated at the hematology institute at Tel-Aviv Soursaky Medical Center and (iv) underwent whole-body $\left[{ }^{18} \mathrm{~F}\right]$ FDG PET-CT in our department before and/or after CAR-T transfusion. 
A total of 138 PET-CT studies of 48 patients treated between April 2019 and April 2021 were identified and included in the study: 30 TD, 42 TT, 44 M1 and 22 M3 PET-CT scans. Thirty-nine (85\%) of the study patients received bridging therapy between TD and TT (33 received systemic and radiation therapy, 6 received systemic therapy, 6 received radiation therapy). M1 and M3 scans were included only if no other anti-lymphoma treatment was added since CAR-T infusion. Median interval between TD and TT studies was 1.4 (IQR 1.3-2) months. Median interval between TT study and CAR-T transfusion was 0.5 (IQR $0.3-$ 1.2) months. Median time interval between CAR-T transfusion and the next PET-CT studies were 1 (IQR $0.9-1.1)$ and $3(2.8-3.3)$ months for $M 1$ and $M 3$ scans, respectively.

\section{Imaging.}

$\left[{ }^{18}\right.$ F]FDG PET-CT studies were performed on PET-CT scanners (GE Healthcare; DISCOVERY 690 and DISCOVERY Ml; 7 to 8 frames; frame time 1.5-3 minutes) according to our standard protocol with administration of dilute oral contrast agent, injection of $3.7 \mathrm{MBq} / \mathrm{kg}\left[{ }^{18} \mathrm{~F}\right] \mathrm{FDG}$ approximately 60 minutes prior to the study.

For all 138 included studies, SUVmax, TMTV and total lesion glycolysis (TLG) values were documented. SUVmax was measured in the "hottest" nodal or extranodal lymphoma site. Spleen was considered involved if there were focal uptake or diffuse uptake $>150 \%$ of the liver uptake. Bone marrow was considered only in case of focal uptake. TMTV was obtained with the $41 \%$ SUVmax threshold method as recommended by the European Association of Nuclear Medicine [29], by summing the metabolic volumes of all local nodal and extranodal lesions using Q.Volumetrix Al (GE Healthcare). TLG value was computed as the product of measured SUVmean and MTV.

Response assessment was done using two methods: The Deauville five-point scale (1: No uptake; 2 : Uptake $\leq$ mediastinum; 3: Uptake > mediastinum but $\leq$ liver; 4: Moderately increased uptake compared to the liver; 5: Markedly increased uptake compared to the liver and/or new lesions) [9] and $\Delta S U V \max$ method ( $\triangle$ SUVmax was calculated as the percentage change in SUVmax between PET studies) [18]. Deauville score $\leq 3$ and $\Delta S U V \max >66 \%$ were considered favorable response criteria $[9,18]$.

Table 1 summarizes the clinical data and PET-CT findings on TD and TT scans done prior to CAR-T infusion.

\section{Outcome Variables.}

The primary endpoint of the study was overall survival (OS), defined as time from CAR-T transfusion to death from any cause. Both imaging data before and after CAR-T transfusion were evaluated for their role in predicting OS. For imaging data before CAR-T transfusion, secondary endpoints included toxicity (CRS and ICANS, graded according to the American Society for Transplantation and Cellular Therapy criteria [8]) and progression-free survival (PFS), defined as the time from CAR-T transfusion to disease progression as defined by Lugano criteria [9] or to death from any cause. 


\section{Statistical analysis.}

Categorical data were described with contingency tables including frequency and percent. Continuous variables were evaluated for normal distribution and reported as median and interquartile range (IQR). Medians of continuous variables were used as cutoff for defining dichotomous variables (i.e. values above median-positive). Median length of follow-up was observed using reverse censoring method. The median survival time and the probabilities of OS and PFS were estimated using the Kaplan-Meier method. Log-rank test and univariate cox regression were used to study the crude association between studied predictors and OS and PFS. Pearson's $\chi^{2}$ test, Fisher's exact test, and univariate Cox regression were used to study the crude association between the studied predictors and toxicity outcomes. The Mann-Whitney $\mathrm{U}$ test was used to compare medians of continuous variables between two groups. Multivariate cox regression analysis was performed using backward method ( $p>0.1$ was used as criteria for removal) in order to identify independent predictors for OS. A two-sided $P$ value of $<0.05$ was considered as statistically significant. Variables with trend or significant association to OS and PFS, or those known to be of important clinical significance, were tested in the multivariate model. SPSS software (IBM SPSS Statistics for Windows, version 27, IBM corp., Armonk, NY, USA, 2017) was used for statistical analyses. Survival curves were generated using the open-source statistics software R (version 4.0.5, R Foundation for Statistical Computing).

\section{Results}

Patients were followed for a median of 12.8 (IQR 6.4-16.0) months after CAR-T infusion. Median OS was not reached. Six-months and 1-year survival rates were $70.7 \%$ and $52.4 \%$, respectively.

Prediction of survival and toxicity prior to CAR-T infusion

In a univariate Cox regression analysis shown in Table 2, several PET and clinical parameters were found to be significantly predictive of OS and PFS prior to infusion of CAR-T. Patients with TD-SUVmax $>17.1$ and patients with TT-SUVmax $>12.1$ had significantly shorter OS and PFS (see Fig. 1). Higher TT-TMTV and higher TT-TLG were also identified as risk factors for poor OS. Patients with elevated LDH and ECOG performance score $>1$ had significantly shorter OS and PFS.

In a multivariate Cox regression analysis (Table 3) for OS that included age, sex, LDH, ECOG score, TDSUVmax, TT-SUVmax, TT-TMTV and TT-TLG as dichotomous variables, three independent prognostic factor were identified: TD-SUVmax > 17.1 (HR 10.3; 95\% Cl, 2.2-47.7; $\mathrm{PV}<0.01)$, Serum LDH > $450 \mathrm{U} / \mathrm{I}(\mathrm{HR}$ 7.7; 95\% Cl, 1.9-32.0; $\mathrm{Pv}<0.01$ ) and ECOG score > 1 (HR 5.5; 95\% Cl, 1.1-31.0; $\mathrm{Pv}=0.04$ ).

Based on this multivariate model, we assigned a score between 0 and 3 to each of the study patients, based on the number of the independent risk factors that they meet (TD-SUVmax, LDH level and ECOG performance). On Fig. 2, the OS curves of patients with different scores is shown. Patients in our data that met all of the three criteria (assigned a score of 3 ) had median OS of $2.6(95 \% \mathrm{Cl}, 1.1-4.0)$ months. 
Response to bridging therapy as evaluated on the TT scan was not significantly associated with PFS and OS using both the Deauville five-point scale and $\Delta$ SUVmax methods. Only the minority of patients met the favorable response criteria: 7 patients (16.7\%) had Deauville score $\leq 3$, and 2 patients $(6.7 \%)$ had $\Delta$ SUVmax $>66 \%$ on their TT scan.

Among the patients that underwent PET-CT prior to CART transfusion, the post-therapy incidences of anygrade CRS, grade 3-4 CRS and ICANS were 76.2\% (32/42 patients), 11.9\% (5/42 patients) and 21.4\% (9/42 patients), respectively. No statistically significant association was found between TD or TT PET variables and CRS or ICANS in this cohort.

Imaging interpretation of post-CAR-T PET-CT

Response to CAR-T therapy as evaluated on the M1-PET-CT scan was significantly associated with OS using the Deauville five-point scale. While those with Deauville score $>3$ had median survival of $7.9(95 \%$ $\mathrm{Cl}, 3.8-12.0)$ months, median survival of those with Deauville score $\leq 3$ was not reached $(\mathrm{Pv}<0.01)$. While the 1 -year OS for patients with favorable response was $94 \%$, it was $20 \%$ for those with poor response based on the Deauville scale (Fig. 3A).

Using the $\triangle$ SUVmax method, response assessment to CAR-T therapy on the M1-PET-CT scan was not associated with OS when the reference baseline SUVmax was obtained from the TT-PET $\operatorname{scan}(P v=0.38)$ but was significantly associated with OS when the TD-SUVmax was used as the baseline SUVmax (PV = 0.02). Using the $\triangle S U V m a x$ method with TD-SUVmax as reference, the median survival of those who met the favorable criterion was not reached, and those categorized with poor response had median survival of $8.2(95 \% \mathrm{Cl}, 1.3-15.1)$ months ( $\mathrm{Pv}=0.02)$ (Fig. 3B, 3C).

In the group of patients who had a PET-CT scan three months post-CAR-T infusion and were not given any other anti-lymphoma therapy since the CAR-T infusion, response to therapy on the M3 scan was significantly associated with OS using the Deauville five-point scale $(P v<0.01)$. The $\Delta S U V \max$ method could significantly predict OS when the baseline SUVmax was obtained from the TD or TT scan (PV = 0.02 for both, Fig. 4) but not from M1 scan (Pv=0.25).

In multivariate analysis that included the response assessment criteria that were significantly associated with OS on M1 and M3 PET-CT scans, Deauville score > 3 on M1-PET was the only factor significantly associated with OS (HR 7.2; 95\% Cl, 1.5-34.6; Pv =0.01).

\section{Discussion}

The introduction of CAR-T therapy into the clinical practice of R/R DLBCL patients provides a promising approach. However, this novel technology requires an infrastructure, involves complicated logistics, may be associated with severe toxicity, and the response is variable. Thus, it should be considered only in selected patients that may benefit from this therapy. Once CAR-T has been infused, failure should be identified as early as possible to enable change in treatment. 
Serial $\left[{ }^{18} \mathrm{~F}\right]$ FDG PET-CT scans have been included in the imaging algorithm of patients with DLBCL, including a baseline scan performed at diagnosis, scans during and after first-line treatment, as well as when recurrence is suspected [9]. When a PET-CT scan identifies viable R/R DLBCL and the clinician decides that CAR-T is indicated, this study is referred as TD scan. Immediately prior to CAR-T infusion, a TT PET-CT is performed. Some patients receive bridging therapy between TD and TT. For monitoring response to CAR-T therapy, PET-CT is usually performed early (1M scan) and later (3M scan). In the current study we investigated the role of PET-CT performed before CAR-T infusion in predicting outcome thus assisting in selection of patients that may benefit from CAR-T, and the use of post-treatment PET-CT in identifying therapy failure.

Several PET parameters can be used in the clinical practice of reporting PET-CT scans of DLBCL patients. Measurement of SUVmax, comparing SUVmax between different time points (as done using the $\Delta S U V \max$ method), and using the Deauville five-point scale are usually easily performed. Calculation of TMTV and TLG are somewhat more time consuming. In the current study we investigated all the latter parameters on TD, TT, M1 and M3 PET-CT scans.

In a multivariate analysis we performed, the only pre-CAR-T PET parameter found to be an independent risk factor for shorter OS was TD-SUVmax > 17.1. If a patient meets this criterion on TD PET-CT, it appears that already at this point the clinician may suspect that CAR-T might be of a lower added value for the patient. Two pre-treatment clinical parameters were also found to independently predict shorter OS: Serum LDH > 450 U/I (a risk factor identified before by Hirayama et al [30]) and ECOG performance score $>1$. In practice, patients included in our study with all of the three mentioned risk factors had very short OS and probably did not benefit from CAR-T therapy.

Data of recent studies suggested that TMTV measured on TT-PET may be associated with disease progression [19-20]. This finding was validated also in the current study on a longer follow-up period. We also found that reporting TT-SUVmax, which is obtained more easily than TT-TMTV, is enough and provides an alternative practical tool for prediction of survival.

Response to bridging therapy given between TD and TT PET-CT scans assessed by both Deauville scale and $\triangle S U V m a x$ method did not predict post-CAR-T survival. This result highlights again that reporting TDSUVmax and TT-SUVmax should be included in pre-treatment PET-CT reports rather than focusing on response assessment to the bridging therapy.

Although other small studies found an association between TT-PET and CRS [20, 23], we did not find association between PET variables at TD or TT and toxicity, possibly because of high prevalence of CRS and small numbers of high-grade CRS and ICANS.

Monitoring response to CAR-T therapy is essential in order to identify CAR-T failure as early as possible. A practical tool that separates correctly between responders with longer predicted OS and non-responders with shorter predicted OS is of great importance. On M1-PET-CT scan, Deauville score $>3$ was found in our study as the strongest predictor of short OS. $\triangle$ SUVmax measurement on M1-PET was also found of 
predictive value when comparing M1-SUVmax to TD-SUVmax (and not to TT-SUVmax). Either of these parameters on M1-PET-CT report may provide critical information to clinicians and guide clinical decisions.

On M3-PET-CT scan, our findings suggest that response assessment identified correctly patients with favorable response and longer OS using both Deauville scale and $\triangle S U V \max$ method (with TD-SUVmax or TT-SUVmax, but not M1-SUVmax as baseline values). Using either method in the current study, none of the patients identified with favorable response on M3-PET-CT died during their follow-up. Since we only included patients not given any anti-lymphoma treatment between CAR-T infusion and M3-PET-CT scan, the number of patients included in this analysis was small and their follow-up time was limited. This limitation applies for this study in general, and further validation of our results on larger cohorts with longer follow-up is needed.

\section{Conclusions}

Our results suggest that measurement of SUVmax of the "hottest" lymphoma site on pre-treatment PETCT, mainly in combination with serum LDH levels and evaluation of ECOG performance status, may separate between R/R DLBCL patients that may benefit from CAR-T therapy and those with poor prognosis in whom this treatment is less valuable. Post-CAR-T PET-CT may identify early therapy failure. One month after CAR-T infusion, Deauville score and $\triangle$ SUVmax measurement using TD-SUVmax as the reference for comparison, can both be used for separating responders and non-responders.

\section{Abbreviations}

R/R DLBCL: relapsed/refractory diffuse large B-cell lymphoma

CD19: Cluster of differentiation 19

CAR-T: Chimeric antigen receptor T-cell

CRS: Cytokine release syndrome

ICANS: Immune effector cell-associated neurotoxicity syndrome

ECOG: Eastern Cooperative Oncology Group

GCB: Germinal center B-Cell like

COO: Cell of origin

$\left[{ }^{18} \mathrm{~F}\right] \mathrm{FDG}:{ }^{18} \mathrm{~F}$-fluorodeoxyglucose

PET-CT: Positron emission tomography - computed tomography 
TD: Time of decision

TT: Time of transfusion

M1: one month

M3: three months

SUVmax: Maximum standardized uptake value

SUVmean: Mean standardized uptake value

TMTV: Total metabolic tumor volume

TLG: Total lesion glycolysis

OS: Overall survival

PFS: Progression free survival

IQR: Interquartile range

Pv: P-Value

Cl: Confidence interval

HR: Hazard ratio

\section{Declarations}

\section{Declarations/ Competing interests}

All authors have no relevant financial or non-financial interests to disclose and have no conflicts of interest to declare that are relevant to the content of this article.

\section{Acknowledgements}

None.

\section{Funding}

No funding was received.

\section{Availability of data and materials}

The datasets used and/or analyzed during the current study are available from the corresponding author on reasonable request. 


\section{Ethics approval and consent to participate}

All procedures performed in studies involving human participants were in accordance with the ethical standards of the institutional and/or national research committee and with the 1964 Helsinki Declaration and its later amendments or comparable ethical standards. All included data were collected as part of a retrospective study protocol approved by the local institutional ethics committee, which waived written informed consent (Reference ID 0503-20-TLV).

\section{Consent for publication}

Not applicable.

\section{References}

1. Van Den Neste E, Schmitz N, Mounier N, Gill D, Linch D, Trneny M, Milpied N, Radford J, Ketterer N, Shpilberg O, Dührsen U. Outcome of patients with relapsed diffuse large B-cell lymphoma who fail second-line salvage regimens in the International CORAL study. Bone marrow transplantation. 2016 Jan;51(1):51-7. https://doi.org/10.1038/bmt.2015.213

2. Crump M, Neelapu SS, Farooq U, Van Den Neste E, Kuruvilla J, Westin J, Link BK, Hay A, Cerhan JR, Zhu L, Boussetta S. Outcomes in refractory diffuse large B-cell lymphoma: results from the international SCHOLAR-1 study. Blood, The Journal of the American Society of Hematology. 2017 Oct 19;130(16):1800-8. https://doi.org/10.1182/blood-2017-03-769620

3. Locke FL, Ghobadi A, Jacobson CA, Miklos DB, Lekakis LJ, Oluwole OO, Lin Y, Braunschweig I, Hill BT, Timmerman JM, Deol A. Long-term safety and activity of axicabtagene ciloleucel in refractory large B-cell lymphoma (ZUMA-1): a single-arm, multicentre, phase 1-2 trial. The lancet oncology. 2019 Jan 1;20(1):31-42. https://doi.org/10.1016/S1470-2045(18)30864-7

4. Neelapu SS, Locke FL, Bartlett NL, Lekakis LJ, Miklos DB, Jacobson CA, Braunschweig I, Oluwole 00, Siddiqi T, Lin Y, Timmerman JM. Axicabtagene ciloleucel CAR T-cell therapy in refractory large B-cell lymphoma. New England Journal of Medicine. 2017 Dec 28;377(26):2531-44. https://doi.org/10.1056/NEJMoa1707447

5. Schuster SJ, Bishop MR, Tam CS, Waller EK, Borchmann P, McGuirk JP, Jäger U, Jaglowski S, Andreadis C, Westin JR, Fleury I. Tisagenlecleucel in adult relapsed or refractory diffuse large B-cell lymphoma. New England Journal of Medicine. 2019 Jan 3;380(1):45-56. https://doi.org/10.1056/NEJMoa1804980

6. Neelapu SS. Managing the toxicities of car T-cell therapy. Hematological oncology. 2019 Jun;37:4852. https://doi.org/10.1002/hon.2595

7. Sievers S, Watson G, Johncy S, Adkins S. Recognizing and grading CAR T-cell toxicities: an advanced practitioner perspective. Frontiers in Oncology. 2020 Jun 24;10:885. https://doi.org/10.3389/fonc. 2020.00885 
8. Lee DW, Santomasso BD, Locke FL, Ghobadi A, Turtle CJ, Brudno JN, Maus MV, Park JH, Mead E, Pavletic S, Go WY. ASTCT consensus grading for cytokine release syndrome and neurologic toxicity associated with immune effector cells. Biology of Blood and Marrow Transplantation. 2019 Apr 1;25(4):625-38. https://doi.org/10.1016/j.bbmt.2018.12.758

9. Cheson BD, Fisher RI, Barrington SF, Cavalli F, Schwartz LH, Zucca E, Lister TA. Recommendations for initial evaluation, staging, and response assessment of Hodgkin and non-Hodgkin lymphoma: the Lugano classification. Journal of clinical oncology. 2014 Sep 20;32(27):3059. https://doi.org/10.1200/JC0.2013.54.8800

10. Dührsen U, Müller S, Hertenstein B, Thomssen H, rg Kotzerke J, Mesters R, Berdel WE, Franzius C, Kroschinsky F, Weckesser M, Kofahl-Krause D. Positron emission tomography-guided therapy of aggressive non-Hodgkin lymphomas (PETAL): a multicenter, randomized phase III trial. Journal of Clinical Oncology. 2018;36(20):2024-34. https://doi.org/10.1200/JC0.2017.76.8093

11. Burggraaff CN, de Jong A, Hoekstra OS, Hoetjes NJ, Nievelstein RA, Jansma EP, Heymans MW, de Vet $\mathrm{HC}$, Zijlstra JM. Predictive value of interim positron emission tomography in diffuse large B-cell lymphoma: a systematic review and meta-analysis. European journal of nuclear medicine and molecular imaging. 2019 Jan;46(1):65-79. https://doi.org/10.1007/s00259-018-4103-3

12. Cashen AF, Dehdashti F, Luo J, Homb A, Siegel BA, Bartlett NL. 18F-FDG PET/CT for early response assessment in diffuse large B-cell lymphoma: poor predictive value of international harmonization project interpretation. Journal of Nuclear Medicine. 2011 Mar 1;52(3):386-92. https://doi.org/10.2967/jnumed.110.082586

13. Pregno P, Chiappella A, Bello M, Botto B, Ferrero S, Franceschetti S, Giunta F, Ladetto M, Limerutti G, Menga M, Nicolosi M. Interim 18-FDG-PET/CT failed to predict the outcome in diffuse large B-cell lymphoma patients treated at the diagnosis with rituximab-CHOP. Blood, The Journal of the American Society of Hematology. 2012 Mar 1;119(9):2066-73. https://doi.org/10.1182/blood-201106-359943

14. González-Barca E, Canales M, Cortés M, Vidal MJ, Salar A, Oriol A, Bargay J, Bello JL, Sánchez JJ, Tomás JF, Donato E. Predictive value of interim 18F-FDG-PET/CT for event-free survival in patients with diffuse large B-cell lymphoma homogenously treated in a phase II trial with six cycles of RCHOP-14 plus pegfilgrastim as first-line treatment. Nuclear medicine communications. 2013 Oct 1;34(10):946-52. https://doi.org/10.1097/MNM.0b013e328363c695

15. Zhu Y, Lu J, Wei X, Song S, Huang G. The predictive value of interim and final [18F] fluorodeoxyglucose positron emission tomography after rituximab-chemotherapy in the treatment of non-Hodgkin's lymphoma: a meta-analysis. BioMed research international. 2013 Jan 1;2013. https://doi.org/10.1155/2013/275805

16. Mamot C, Klingbiel D, Hitz F, Renner C, Pabst T, Driessen C, Mey U, Pless M, Bargetzi M, Krasniqi F, Gigli F. Final results of a prospective evaluation of the predictive value of interim positron emission tomography in patients with diffuse large B-cell lymphoma treated with R-CHOP-14 (SAKK 38/07). Journal of clinical oncology. 2015 Aug 10;33(23):2523-9. https://doi.org/10.1200/JC0.2014.58.9846 
17. Kostakoglu L, Martelli M, Sehn LH, Belada D, Carella AM, Chua N, Gonzalez-Barca E, Hong X, Pinto A, Shi Y, Tatsumi Y. End-of-treatment PET/CT predicts PFS and OS in DLBCL after first-line treatment: results from GOYA. Blood advances. 2021 Mar 9;5(5):1283-90.

https://doi.org/10.1182/bloodadvances.2020002690

18. Rekowski J, Hüttmann A, Schmitz C, Müller SP, Kurch L, Kotzerke J, Franzius C, Weckesser M, Bengel FM, Freesmeyer M, Hertel A. Interim PET evaluation in diffuse large B-cell lymphoma employing published recommendations: Comparison of the Deauville 5-point scale and the $\Delta S U V \max$ method. Journal of Nuclear Medicine. 2020 May 1. https://doi.org/10.2967/jnumed.120.244145

19. Vercellino L, Di Blasi R, Kanoun S, Tessoulin B, Rossi C, D'Aveni-Piney M, Obéric L, Bodet-Milin C, Bories P, Olivier P, Lafon I. Predictive factors of early progression after CAR T-cell therapy in relapsed/refractory diffuse large B-cell lymphoma. Blood advances. 2020 Nov 24;4(22):5607-15. https://doi.org/10.1182/bloodadvances.2020003001

20. Wang J, Hu Y, Yang S, Wei G, Zhao X, Wu W, Zhang Y, Zhang Y, Chen D, Wu Z, Xiao L. Role of fluorodeoxyglucose positron emission tomography/computed tomography in predicting the adverse effects of chimeric antigen receptor T cell therapy in patients with non-Hodgkin lymphoma. Biology of Blood and Marrow Transplantation. 2019 Jun 1;25(6):1092-8.

https://doi.org/10.1016/j.bbmt.2019.02.008

21. Iacoboni G, Simó M, Villacampa G, Catalá E, Carpio C, Díaz-Lagares C, Vidal-Jordana Á, Bobillo S, Marín-Niebla A, Pérez A, Jiménez M. Prognostic impact of total metabolic tumor volume in large Bcell lymphoma patients receiving CAR T-cell therapy. Annals of Hematology. $2021 \mathrm{Jul}$ 8:1-8. https://doi.org/10.1007/s00277-021-04560-6

22. Shah NN, Nagle SJ, Torigian DA, Farwell MD, Hwang WT, Frey N, Nasta SD, Landsburg D, Mato A, June $\mathrm{CH}$, Schuster SJ. Early positron emission tomography/computed tomography as a predictor of response after CTL019 chimeric antigen receptor-T-cell therapy in B-cell non-Hodgkin lymphomas. Cytotherapy. 2018 Dec 1;20(12):1415-8. https://doi.org/10.1016/j.jcyt.2018.10.003

23. Derlin T, Schultze-Florey C, Werner RA, Möhn N, Skripuletz T, David S, Beutel G, Eder M, Ross TL, Bengel FM, Ganser A. 18 F-FDG PET/CT of off-target lymphoid organs in CD19-targeting chimeric antigen receptor T-cell therapy for relapsed or refractory diffuse large B-cell lymphoma. Annals of Nuclear Medicine. 2021 Jan;35(1):132-8. https://doi.org/10.1007/s12149-020-01544-w

24. Meignan M, Gallamini A, Meignan M, Gallamini A, Haioun C. Report on the first international workshop on interim-PET scan in lymphoma. Leukemia \& lymphoma. 2009 Jan 1;50(8):1257-60. https://doi.org/10.1080/10428190903040048

25. Schmitz C, Hüttmann A, Müller SP, Hanoun M, Boellaard R, Brinkmann M, Jöckel KH, Dührsen U, Rekowski J. Dynamic risk assessment based on positron emission tomography scanning in diffuse large B-cell lymphoma: post-hoc analysis from the PETAL trial. European Journal of Cancer. 2020 Jan 1;124:25-36. https://doi.org/10.1016/j.ejca.2019.09.027

26. Casasnovas RO, Meignan M, Berriolo-Riedinger A, Bardet S, Julian A, Thieblemont C, Vera P, Bologna $S$, Brière J, Jais JP, Haioun C. SUVmax reduction improves early prognosis value of interim positron 
emission tomography scans in diffuse large B-cell lymphoma. Blood, The Journal of the American Society of Hematology. 2011 Jul 7;118(1):37-43. https://doi.org/10.1182/blood-2010-12-327767

27. Toledano MN, Vera P, Tilly H, Jardin F, Becker S. Comparison of therapeutic evaluation criteria in FDG$\mathrm{PET} / \mathrm{CT}$ in patients with diffuse large-cell B-cell lymphoma: prognostic impact of tumor/liver ratio. PLoS One. 2019 Feb 7;14(2):e0211649. https://doi.org/10.1371/journal.pone.0211649

28. Li X, Sun X, Li J, Liu Z, Mi M, Zhu F, Wu G, Lan X, Zhang L. Interim PET/CT based on visual and semiquantitative analysis predicts survival in patients with diffuse large B-cell lymphoma. Cancer medicine. 2019 Sep;8(11):5012-22. https://doi.org/10.1002/cam4.2404

29. Boellaard R, Delgado-Bolton R, Oyen WJ, Giammarile F, Tatsch K, Eschner W, Verzijlbergen FJ, Barrington SF, Pike LC, Weber WA, Stroobants S. FDG PET/CT: EANM procedure guidelines for tumour imaging: version 2.0. European journal of nuclear medicine and molecular imaging. 2015 Feb;42(2):328-54. https://doi.org/10.1007/s00259-014-2961-x

30. Hirayama AV, Gauthier J, Hay KA, Voutsinas JM, Wu Q, Gooley T, Li D, Cherian S, Chen X, Pender BS, Hawkins RM. The response to lymphodepletion impacts PFS in patients with aggressive nonHodgkin lymphoma treated with CD19 CAR T cells. Blood, The Journal of the American Society of Hematology. 2019 Apr 25;133(17):1876-87. https://doi.org/10.1182/blood-2018-11-887067

\section{Tables}


Table 1

Patients characteristics prior to CAR-T infusion

\begin{tabular}{|c|c|c|c|}
\hline & & & All patients $(n=48)$ \\
\hline \multirow[t]{9}{*}{ Clinical characteristics } & Age (years) & (median, IQR) & $68(61-76)$ \\
\hline & Gender & (Male, $\mathrm{N}, \%)$ & $25(52 \%)$ \\
\hline & Serum LDH $(\mathrm{U} / \mathrm{I})$ & (median, IQR) & $445(356-575)$ \\
\hline & ECOG performance & (score $>1, N, \%)$ & $25(53 \%)$ \\
\hline & De novo vs transformed & (De novo, $\mathrm{N}, \%$ ) & $14(30 \%)$ \\
\hline & $\mathrm{COO}$ & (GCB, N, \%) & $20(42 \%)$ \\
\hline & Number of prior lines & (median, range) & $2(2-4)$ \\
\hline & Bridging therapy & (yes, $N, \%)$ & $39(85 \%)$ \\
\hline & CAR-T product & (Axi-cel, N, \%) & $34(72 \%)$ \\
\hline \multirow[t]{4}{*}{ TD-PET parameters } & TD-SUVmax & (median, IQR) & $17.1(11.5-23.5)$ \\
\hline & TD-TMTV (ml) & (median, IQR) & $17.4(7.3-46.3)$ \\
\hline & TD-TLG (g) & (median, IQR) & $216.8(51.1-432.7)$ \\
\hline & TD-Deauville & (score $>3, \mathrm{~N}, \%$ ) & $30(100 \%)$ \\
\hline \multirow[t]{5}{*}{ TT-PET parameters } & TT-SUVmax & (median, IQR) & $12.1(7.5-19.5)$ \\
\hline & TT-TMTV (ml) & (median, IQR) & $13.5(2.6-61.9)$ \\
\hline & TT-TLG (g) & (median, IQR) & 70.5 (19.3-493.6) \\
\hline & TT-Deauville & (score $>3, N, \%)$ & 35 (83\%) \\
\hline & $\Delta \mathrm{SUVmax}(\mathrm{TD} \rightarrow \mathrm{TT})$ & $(\Delta>66 \%, N, \%)$ & $28(93 \%)$ \\
\hline
\end{tabular}

TD-PET, time of decision PET, the scan based on which CAR-T was selected as the next line of therapy; TT-PET, time of transfusion PET, the scan performed immediately prior to CAR-T transfusion; LDH, lactate dehydrogenase; ECOG, Eastern Cooperative Oncology Group; Transformed, DLBCL transformed from another lymphoma; $\mathrm{COO}$, cell of origin; GCB, germinal center B-Cell like; Bridging therapy, anti-lymphoma treatment given between TD and TT; CAR-T product, chimeric antigen receptor T-cell product (Axi-cel or Tisa-cel); SUVmax, maximum standardized uptake value; TMTV, total metabolic tumor volume; TLG, total lesion glycolysis. 
Table 2

Univariate analysis of pre-CAR-T clinical and PET factors for overall survival (OS) and for progression free survival (PFS)

\begin{tabular}{|c|c|c|c|c|c|}
\hline & & \multicolumn{2}{|l|}{ OS } & \multicolumn{2}{|l|}{ PFS } \\
\hline & & $\mathrm{Pv}$ & $\mathrm{HR}(95 \% \mathrm{Cl})$ & $\mathrm{Pv}$ & $\mathrm{HR}(95 \% \mathrm{Cl})$ \\
\hline \multirow[t]{11}{*}{ Clinical data } & Age & 0.89 & & 0.67 & \\
\hline & Age $>70$ years & 0.99 & & 0.94 & \\
\hline & Gender: male (vs female) & 0.10 & & 0.66 & \\
\hline & Serum LDH & $<.01 *$ & $\begin{array}{l}1.001(1.001- \\
1.002)\end{array}$ & $<.01 *$ & $\begin{array}{l}1.001(1.000- \\
1.001)\end{array}$ \\
\hline & Serum LDH > $450 \mathrm{U} / \mathrm{I}$ & $<.01 *$ & $8.5(2.6-27.2)$ & $<.01 *$ & $3.3(1.5-7.5)$ \\
\hline & $\begin{array}{l}\text { ECOG performance score > } \\
1\end{array}$ & $0.01 *$ & $5.1(1.5-17.8)$ & $0.03^{*}$ & $2.5(1.1-5.8)$ \\
\hline & De novo (vs transformed) & 0.42 & & 0.10 & \\
\hline & COO: GCB (vs non-GCB) & 0.87 & & 0.19 & \\
\hline & Number of prior lines $>2$ & 0.37 & & 0.64 & \\
\hline & Bridging therapy (vs no) & 0.72 & & 0.30 & \\
\hline & $\begin{array}{l}\text { CAR-T product: Axi-cel (vs } \\
\text { Tisa-cel) }\end{array}$ & 0.27 & & 0.44 & \\
\hline \multirow[t]{6}{*}{ TD-PET data } & TD-SUVmax & 0.06 & & 0.07 & \\
\hline & TD-SUVmax > 17.1 & $0.02 *$ & $4.8(1.3-18.1)$ & $0.04 *$ & $2.5(1.1-6.1)$ \\
\hline & TD-TMTV & 0.84 & & 0.61 & \\
\hline & TD-TMTV > $17.4(\mathrm{ml})$ & 0.63 & & 0.12 & \\
\hline & TD-TLG & 0.84 & & 0.83 & \\
\hline & TD-TLG > $216.8(\mathrm{~g})$ & 0.19 & & 0.14 & \\
\hline \multirow[t]{5}{*}{ TT-PET data } & TT-SUVmax & $0.02 *$ & $\begin{array}{l}1.062(1.010- \\
1.116)\end{array}$ & $0.01 *$ & $\begin{array}{l}1.058(1.016- \\
1.102)\end{array}$ \\
\hline & TT-SUVmax > 12.1 & $0.02 *$ & $3.7(1.3-11.3)$ & $0.01 *$ & $3.1(1.4-7.1)$ \\
\hline & TT-TMTV & $0.02 *$ & $\begin{array}{l}1.001(1.000- \\
1.002)\end{array}$ & 0.33 & \\
\hline & TT-TMTV > $13.5(\mathrm{ml})$ & 0.07 & & 0.06 & \\
\hline & TT-TLG & $0.01 *$ & $\begin{array}{l}1.000(1.000- \\
1.000)\end{array}$ & 0.13 & \\
\hline
\end{tabular}




\begin{tabular}{|llll|}
\hline & & OS & PFS \\
\hline & TT-TLG $>70.5(\mathrm{~g})$ & 0.07 & 0.06 \\
\hline $\begin{array}{l}\text { Response } \\
\text { criteria }\end{array}$ & TT-Deauville $>3$ & 0.25 & 0.33 \\
\cline { 2 - 4 } & $\Delta$ SUVmax $(\mathrm{TD} \rightarrow \mathrm{TT})>66 \%$ & 0.39 & 0.21 \\
\hline
\end{tabular}

Each clinical and PET parameter known before CAR-T infusion was analyzed on a univariate Cox regression for OS and for PFS. Continuous variables were analyzed also as dichotomous variables, using commonly used previously defined cut-offs (age > 70, LDH > $450 \mathrm{U} / \mathrm{I}$ ) or median as cut-offs. The hazard ratio $(\mathrm{HR})$ with $95 \%$ confidence interval $(\mathrm{Cl})$ is presented for variables found significantly associated $(\mathrm{Pv}$ $<0.05$ ) with overall survival (OS) or with progression free survival (PFS).

Table 3

Multivariate analysis for overall survival (OS) - A model for patient selection for CAR-T therapy

\begin{tabular}{|lll|}
\hline Variables & HR $(95 \%$ Cl $)$ & Pv \\
\hline Serum LDH $>450 \mathrm{U} / \mathrm{I}$ & $7.7(1.9-32.0)$ & $<0.01$ \\
\hline ECOG performance score $>1$ & $5.5(1.1-31.0)$ & 0.04 \\
\hline TD-SUVmax $>17.1$ & $10.3(2.2-47.7)$ & $<0.01$ \\
\hline
\end{tabular}

On a multivariate Cox regression for OS, the presented dichotomous variables were identified as an independent risk factors for shorter OS. Their hazard ratio (HR) with $95 \%$ confidence interval $(\mathrm{Cl})$ is presented.

\section{Figures}




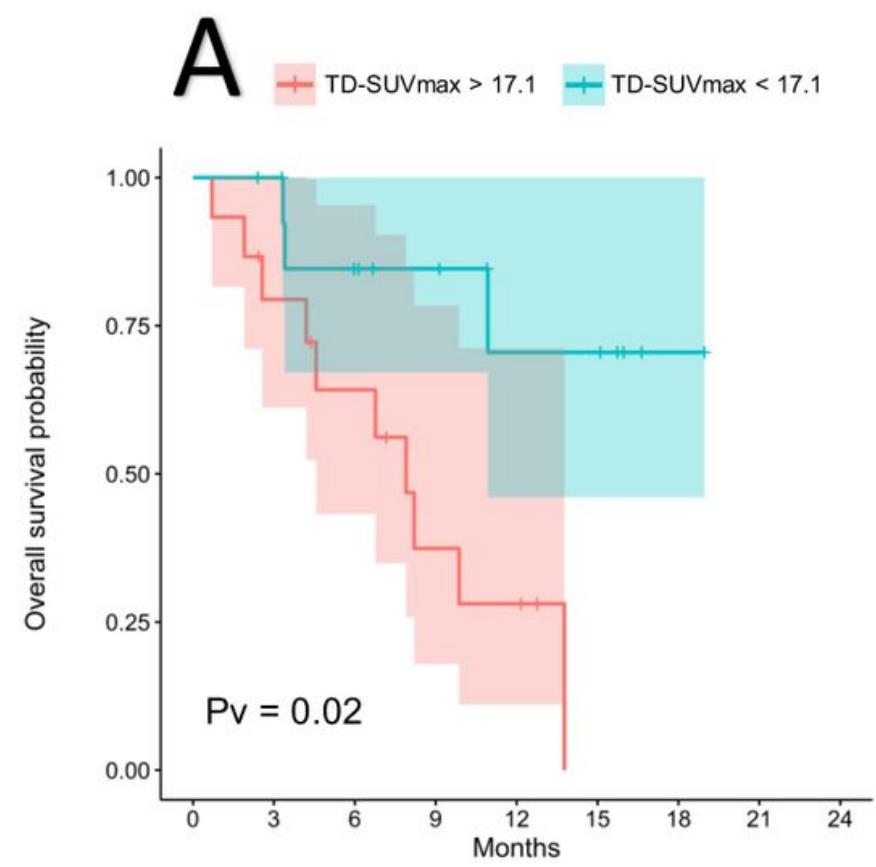

Number at risk

$+\left\{\begin{array}{ccccccccc}15 & 11 & 8 & 4 & 3 & 0 & 0 & 0 & 0 \\ 15 & 14 & 10 & 8 & 5 & 5 & 1 & 0 & 0 \\ \hline 0 & 3 & 6 & 9 & \begin{array}{c}12 \\ \text { Months }\end{array} & 15 & 18 & 21 & 24\end{array}\right.$
B

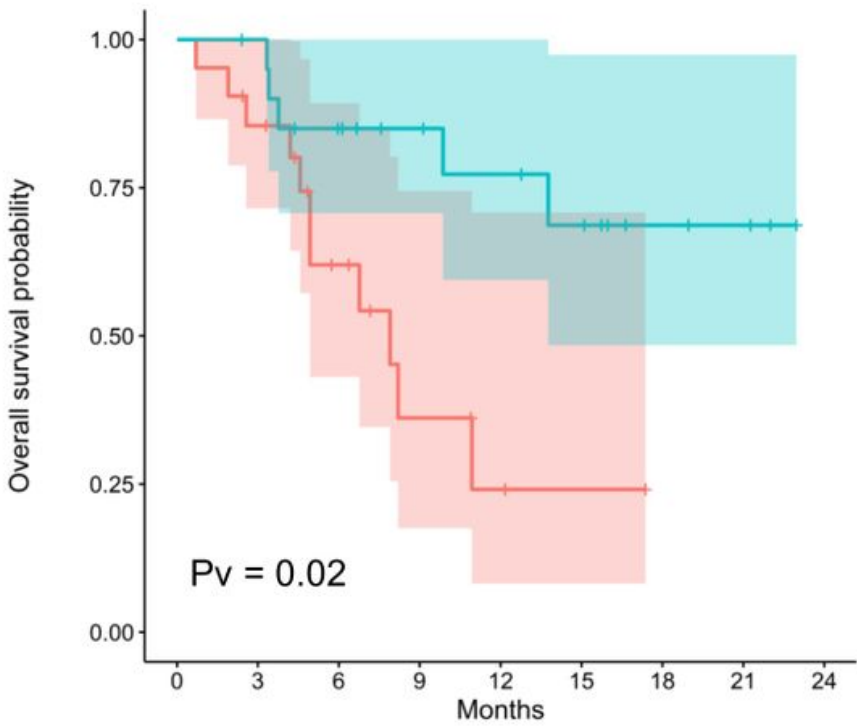

Number at risk

$+$\begin{tabular}{ccccccccc}
21 & 17 & 9 & 4 & 2 & 1 & 0 & 0 & 0 \\
21 & 20 & 15 & 12 & 10 & 8 & 4 & 3 & 0 \\
\hline 0 & 3 & 6 & 9 & $\begin{array}{c}12 \\
\text { Months }\end{array}$ & 15 & 18 & 21 & 24
\end{tabular}

\section{Figure 1}

OS prediction based on TD-SUVmax and TT-SUVmax. Note the significant shorter OS of those with higher TD-SUVmax (A) and those with higher TT-SUVmax (B). 


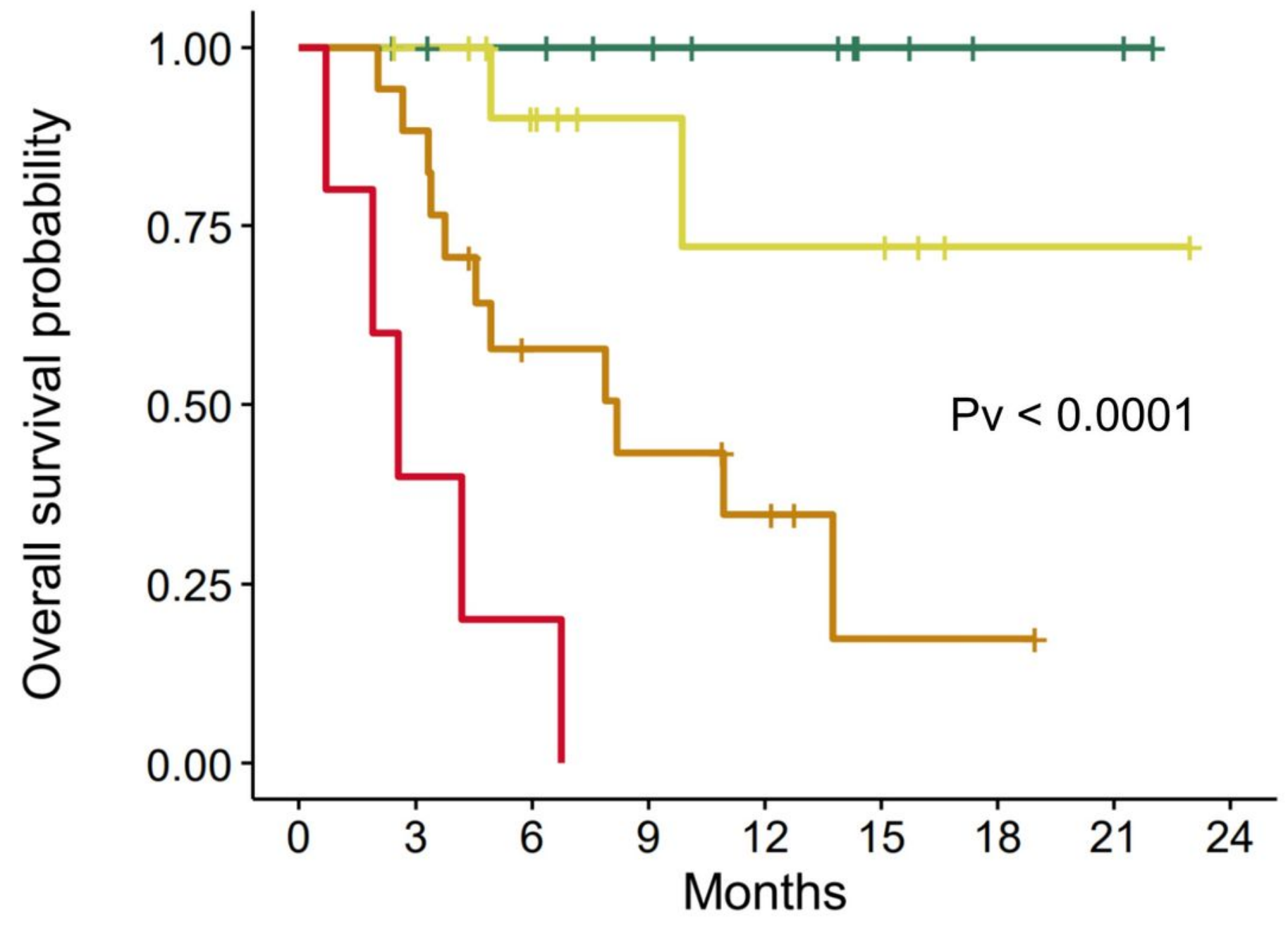

\section{Number at risk}

\begin{tabular}{c|ccccccccc}
+ Score 0 & 13 & 12 & 11 & 9 & 7 & 4 & 2 & 2 & 0 \\
+ Score 1- & 13 & 12 & 8 & 5 & 4 & 4 & 1 & 1 & 0 \\
+ Score 2 & -17 & 15 & 8 & 6 & 4 & 1 & 1 & 0 & 0 \\
+ Score 3- & -5 & 2 & 1 & 0 & 0 & 0 & 0 & 0 & 0 \\
\hline & 0 & 3 & 6 & 9 & 12 & 15 & 18 & 21 & 24 \\
Months & & & 1
\end{tabular}

Figure 2

OS prediction based on the model for patient selection presented on Table 3. Each of the study patients was assigned a score between 0 and 3 based on the number of the independent risk factors they have (TD-SUVmax > 17.1, LDH > $450 \mathrm{U} / \mathrm{I}$ and ECOG performance > 1). Note the significant different OS probability curves between patients with different scores. 


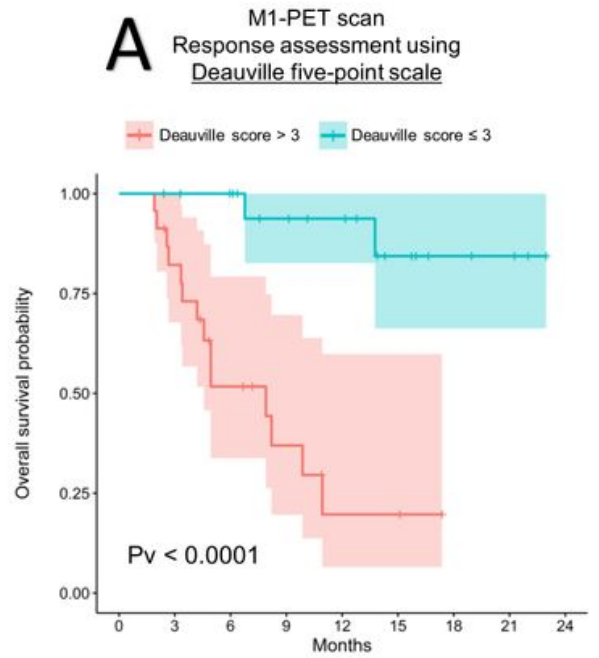

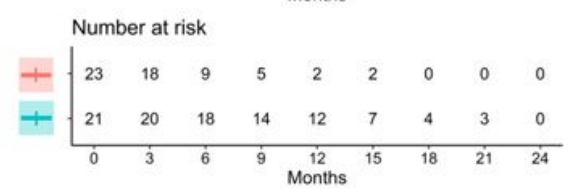

B $\begin{gathered}\text { M1-PET scan } \\ \text { Response assessment using } \\ \triangle S U V \text { max method (TT } \rightarrow M 1)\end{gathered}$
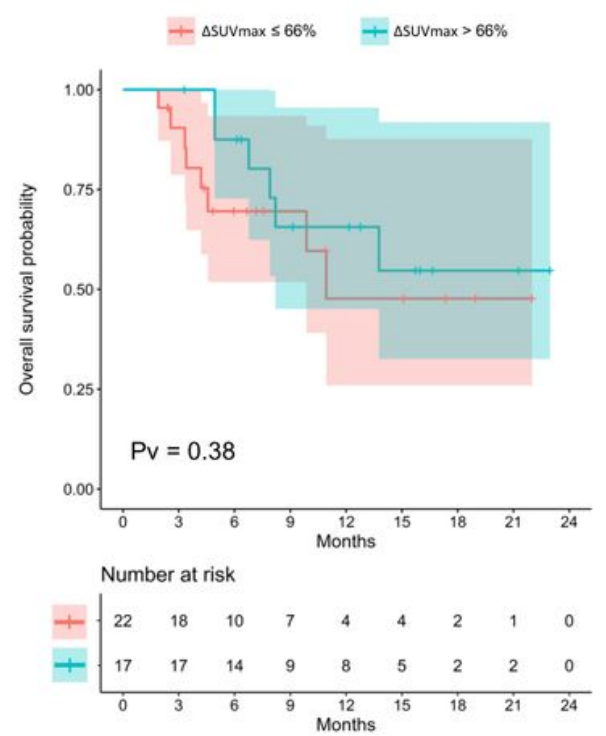

$C \begin{gathered}\text { M1-PET scan } \\ \text { Response assessment using } \\ \Delta \text { SUVmax method (TD } \rightarrow M 1 \text { ) }\end{gathered}$

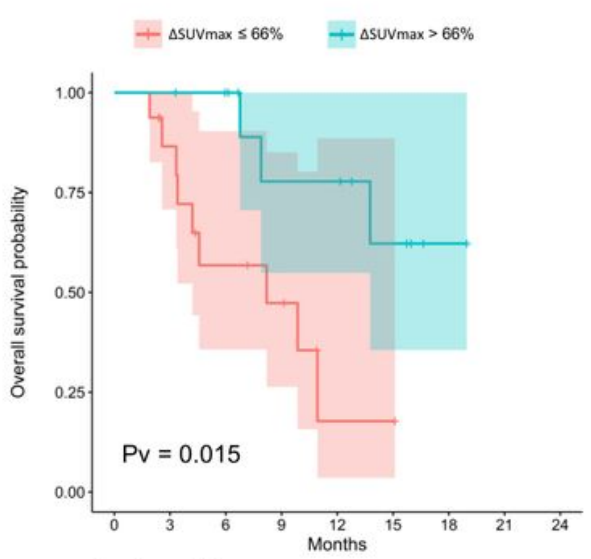

$+\underbrace{\text { Number at risk }}_{0}$\begin{tabular}{ccccccccc}
16 & 12 & 7 & 5 & 1 & 1 & 0 & 0 & 0 \\
13 & 13 & 11 & 7 & 7 & 4 & 1 & 0 & 0 \\
\hline 0 & 3 & 6 & 9 & $\begin{array}{c}12 \\
\text { Months }\end{array}$ & 15 & 18 & 21 & 24
\end{tabular}

Figure 3

OS prediction based on different response assessment criteria on M1-PET. Note the significant short OS of those with Deauville score $>3$ on M1-PET (A). Using the $\triangle$ SUV method, when TT-SUVmax was used as the baseline value, the criterion of $\Delta S U V \max <66 \%$ failed to predict OS in our cohort (B). However, when TD-SUVmax was used as the baseline value, those with $\Delta$ SUVmax $<66 \%$ had significant shorter OS (C) and this method could early define CAR-T failure.
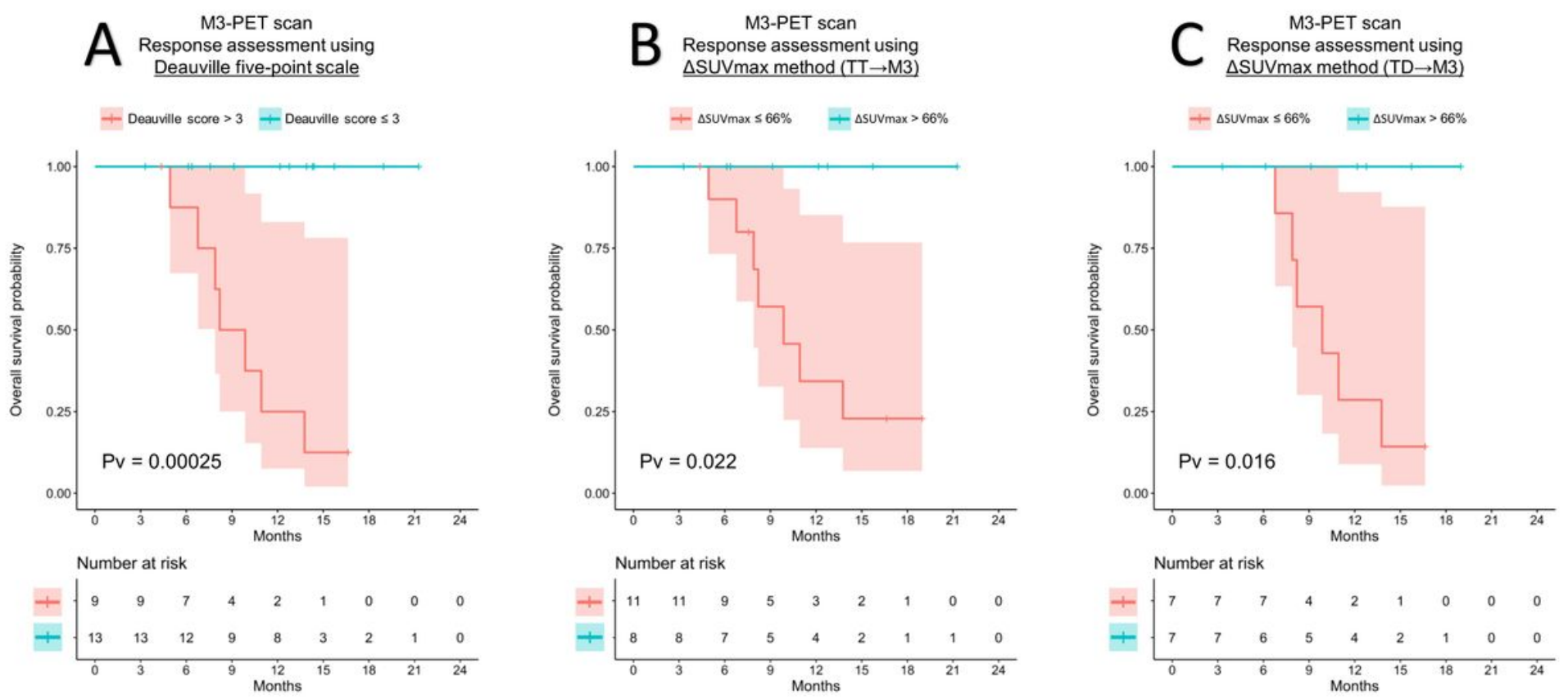

\section{Figure 4}


OS prediction based on different response assessment criteria on M3-PET. Deauville score $\leq 3(\mathrm{~A})$, $\Delta$ SUVmax $(T T \rightarrow M 3)>66 \%(B)$ and $\Delta$ SUVmax $(T T \rightarrow M 3)>66 \%$ (C) could all identify on M3-PET patients with significant longer OS. 